\title{
Phosphate group adsorption capacity of inorganic elements affects bond strength between CAD/CAM composite block and luting agent
}

\author{
Eri TOKUNAGA ${ }^{1}$, Noriyuki NAGAOKA², Yukinori MARUO ${ }^{3}$, Kumiko YOSHIHARA4, Goro NISHIGAWA ${ }^{3}$ \\ and Shogo MINAGI ${ }^{1}$
${ }^{1}$ Department of Occlusal and Oral Functional Rehabilitation, Graduate School of Medicine, Dentistry and Pharmaceutical Sciences, Okayama University, 2-5-1 Shikata-cho, Kita-ku, Okayama 700-8525, Japan Japan
Corresponding author, Eri TOKUNAGA; E-mail: tokunaga.eri@s.okayama-u.ac.jp \\ ${ }^{2}$ Advanced Research Center for Oral and Craniofacial Science, Okayama University, 2-5-1 Shikata-cho, Okayama 700-8525, Japan \\ ${ }^{3}$ Department of Occlusion and Removable Prosthodontics, Okayama University, 2-5-1 Shikata-cho, Okayama 700-8525, Japan \\ ${ }^{4}$ Health Research Institute, National Institute of Advanced Industrial Science and Technology, 2217-14 Hayashi-cho, Takamatsu, Kagawa 761-0395,
}

\begin{abstract}
This study aimed to investigate whether inorganic elements of polymer-infiltrated ceramic (PIC) and microfilled resin (MFR) for CAD/ CAM would affect initial bond strength to luting agent. Inorganic elements of PIC and MFR were different with shape and ingredient observed by SEM, STEM and EDS. Microtensile bond strengths ( $\mu$ TBS) value of PIC was increased by 10-methacryloyloxydecyl dihydrogen phosphate (MDP) and acetic acid (AA)- or MDP-activated silane treatment, and further increased by succeeding heat treatment (HT). The $\mu$ TBS of MFR was increased by MDP and MDP-activated silane, but decreased by AA-activated silane without HT. The HT improved the $\mu$ TBS of MFR with AA-activated silane, but conversely for MDP-activated silane. Only in MFR, phosphoric acid (PA) application before each surface treatment dramatically decreased the $\mu$ TBS of AA-activated silane. FTIR peaks in MFR shifted according to phosphate group's peak. MFR would possess high phosphate group adsorption capacity, with MDP effectively improving its bonding capability.
\end{abstract}

Keywords: Microtensile bond strength, Polymer-infiltrated ceramic, Microfilled resins, Methacryloyloxydecyl dihydrogen phosphate (MDP), Silane

\section{INTRODUCTION}

Ceramic restorations fabricated using CAD/CAM technology are an answer to a slew of expectations and concerns: patient's expectation for better aesthetic outcome, concerns on metal allergy, biocompatibility and substitution for precious metals in dental alloys ${ }^{1}$. While conventional porcelain materials are composed of silicon dioxide $\left(\mathrm{SiO}_{2}\right), \mathrm{CAD} / \mathrm{CAM}$ restorations have improved mechanical properties due to deposition of high amounts of alumina, zirconia or lithium disilicate. Hence, CAD/ CAM materials have become a choice material in cases that require high mechanical properties such as implant superstructures, long span fixed partial dentures, or Maryland bridges ${ }^{2)}$. Although $\mathrm{CAD} / \mathrm{CAM}$ materials possess higher wear resistance and flexural modulus than enamel or gold alloy, they are inferior in dispersing oral force concentration or overload applied during biting and mastication. Hence, a large impact might have negative impacts on the opposing residual dental structure, the abutment tooth, and the surrounding bone ${ }^{3)}$.

New CAD/CAM composite resin blocks called polymer-infiltrated ceramic (PIC) or microfilled resin (MFR) have been introduced as materials which possess similar flexural strength and wear resistance as the tooth substrates. These blocks also circumvent the problems

Color figures can be viewed in the online issue, which is available at J-STAGE.

Received Jan 27, 2020: Accepted Apr 10, 2020

doi:10.4012/dmj.2020-029 JOI JST.JSTAGE/dmj/2020-029 that have long plagued conventional light-curable composites - lower brittleness, susceptibility to abrasion and attrition, loss of surface lubricity and color stability through long-term use $\mathrm{u}^{4,5)}$ - by employing innovative measures for the resin matrix, filler shape, filler ratio, and blending method ${ }^{6-8)}$. PIC is constructed using a porous ceramic network infiltrated with a methacrylate polymer ${ }^{9}$. MFR consists of nanosized inorganic clusters filled into a resin matrix at high density ${ }^{3,10)}$. These two blocks differ not only in the composition, shape and structure of the inorganic filler material, but also in the composition of the resin matrix. Conventional lightcurable composites reportedly demonstrated about 80-150 MPa of flexural strength and 20-40 HVN of hardness ${ }^{4)}$. As for these new CAD/CAM composite blocks, they demonstrated about $150-200 \mathrm{MPa}$ of flexural strength and 60-160 HVN of hardness ${ }^{5-7)}$.

Higher adhesion between ceramic materials and luting agents plays an important role in preventing stress concentration, debonding and fracture of restorations ${ }^{11)}$. In addition to the mechanical strength of the block itself, the abutment structure and ceramic restoration work as a mass bearing the functional occlusal stresses. To date, surface adhesion is improved by mechanical retention and chemical bonding. Air abrasion creates a micromechanical retentive structure on the adhesive surface and is generally used as a surface pretreatment $\operatorname{method}^{12,13)}$. Hydrofluoric acid etching attacks the inorganic material and produces a topographic pattern 
which facilitates micromechanical retention between luting agents and ceramics ${ }^{13)}$. These treatments reportedly improved the bonding and long-term bond durability of CAD/CAM composites, as well ${ }^{13,14)}$.

Functional monomers promote chemical bonding between the resin matrix and inorganic fillers, and silane coupling agents promote chemical interactions with inorganic elements. 10-methacryloyloxydecyl dihydrogen phosphate (MDP) is an acidic functional monomer that bonds well to numerous materials such as resin, tooth, metal and zirconia. Hence, MDP is widely present in the composition of many commercial primers and self-adhesive luting agents ${ }^{15,16)}$. The structure of MDP molecule consists of a phosphoric acid group at one end, a vinyl group at another end of the molecule, and an ester chain spacer composed of 10 carbons to separate these two active groups. The phosphoric acid group of MDP chemically bonds to hydroxyapatite or metal oxides in alumina and zirconia; the vinyl group facilitates polymerisation with unsaturated carbon bonds in the resin matrix ${ }^{17)}$. Micro-Raman spectroscopy has reportedly confirmed and characterized the chemical bonding of MDP to zirconia ${ }^{15)}$, and that to hydroxyapatite or dentin was observed using carbon-13 nuclear magnetic resonance (NMR) spectroscopy ${ }^{16}$. However, several factors affect the bonding effectiveness of MDP: the optimum concentration of MDP for bonding between dentin and composite resins ${ }^{18)}$, and the influence of solvents and molecular structures of MDP on affinity with zirconia ${ }^{17)}$.

Silane coupling agents have an organofunctional terminal group for bonding to organic resins, and a hydrolysable alkoxyl group for bonding to inorganic elements, as well as a hydrocarbon chain spacer. Hydrolysable alkoxyl group is activated by an acid or base to form silanol. Silanol is attached to the hydroxy group on inorganic substrate surface, producing a strong bond called siloxane bond. Gamma-methacryloxypropyltrimethoxysilane ( $\gamma$-MPTS) is one of the major silane coupling agents widely used in dental field. Commercial silane coupling primers are available in one- or two-bottle type. The two-bottle type is activated by mixing nonhydrolysed silane and an acidic solution of acetic acid or MDP, which is a hydrolysis catalyst, immediately before use. The one-bottle type acts as a silane coupling primer containing pre-hydrolysed silane and an acidic solution, or as a universal adhesive incorporated with functional monomers ${ }^{19,20)}$. Universal adhesives have become widely used because of their convenience and multiuse capability. However, silane coupling monomers in universal adhesives polymerize with each other to form an inactive siloxane oligomer after activation. Hence, one-bottle silane coupling primers or universal primers pose a problem on bonding effectiveness ${ }^{20)}$. Two-bottle silane coupling primers are complex because of multiple bottles and steps, but are deemed more effective than the one-bottle type. On the use of acetic acid and acidic adhesive monomers as a hydrolysis catalyst for silane coupling agents, MDP - compared with acetic acidwas found to increase the bond strength between lithium disilicate ceramic or leucite-reinforced ceramic to luting agent $^{21,22)}$.

High amounts of inorganic elements occupy the surfaces of PIC and $\mathrm{MFR}^{9,10}$. To improve mechanical properties, CAD/CAM composites are also polymerized under high temperature and pressure to minimise interaction between unpolymerized monomers and resins. Nonetheless, to be more effective in increasing the bond strength of these CAD/CAM composite blocks to luting agents, the focus should be on the inorganic elements. Inorganic filler materials used in these new $\mathrm{CAD} / \mathrm{CAM}$ composite blocks are more diverse in their structure, composition, and the ratio of inorganic components exposed on the adhesive interface after milling,5,9,10). Silane coupling agents generally provide effective bonding of inorganic elements to luting agents ${ }^{23)}$. With PIC, shear bond strength to luting agent did not significantly increase by silane coupling treatment ${ }^{24)}$, but by universal adhesives ${ }^{25)}$. A combination of universal adhesives with hydrofluoric acid etching or tribochemical silica coating significantly increased the microtensile strength between PIC and luting agent ${ }^{13)}$. With MFR, treatment with silane coupling agent alone significantly lowered the tensile bond strength when compared with these treatments: MMA-containing resin primer application, air abrasion and silane coupling agent application, or air abrasion and resin primer application $^{12)}$. Hydrofluoric acid and silane coupling agent treatments also significantly increased the shear bond strength between MFR and composite resins ${ }^{14)}$. To date, studies have yielded unclear results and inconsistent findings on the effectiveness of conventional surface treatment methods (such as silane coupling and acid etching with functional monomers) on these CAD/ CAM composite blocks.

In this study, two types of CAD/CAM composite blocks of PIC and MFR with different inorganic filler materials were used. The hypothesis was that the differences in structure, composition and ratio of the inorganic elements in these blocks would not affect the bond strength to luting agent. We also investigated the effectiveness of these surface treatments on the bonding of PIC and MFR to self-adhesive luting agent: (1) silane coupling agent based on $\gamma$-MPTS; (2) MDP as a functional monomer; (3) acetic acid and MDP as hydrolysis catalysts for silane coupling agent.

\section{MATERIALS AND METHODS}

Two CAD/CAM composite blocks of Vita Enamic (Vita Zahnfabrik H. Rauter, Bad Säckingen, Germany) as PIC and Cerasmart 270 (GC, Tokyo, Japan) as MFR were used in this study (Table 1).

\section{Scanning electron microscopy (SEM)}

SEM (JSM-6701F, JEOL, Tokyo, Japan) was used to observe the surface compositions of both CAD/CAM blocks. 1-mm-thick slices were cut from each block using a semi-automated, high-speed, diamond saw (Accutom, Struers, Ballerup, Denmark). The blocks 
Table 1 Materials used in the study

\begin{tabular}{ccc}
\hline Brand & Composition & Manufacturer \\
\hline CAD/CAM composite block & & Monomer \\
& $\begin{array}{c}\text { Filler } \\
(\text { Mass\% }(\text { Vol\%) })\end{array}$ & Mol \\
\hline
\end{tabular}

PIC (polymer infiltrated ceramics)

Vita Enamic Feldspar ceramic enriched with aluminum oxide (86 (75))
UDMA, TEGDMA

Vita Zahnfabrik H.Rauter, Bad Säckingen, Germany

MFR (microfilled resins)
Cerasmart 270
Silica (20 nm), Barium glass (300 nm) (71)
Bis-MEPP, UDMA, DMA
GC, Tokyo, Japan

Luting agent

Paste A: Bis-GMA, TEGDMA,

Hydrophobic aromatic

Silanated barium glass filler, Silanated fluoroalminosilicate Panavia V5 glass filler, Colloidal silica, Silanated alminium oxide filler, Particle size: 0.01-12 $\mu \mathrm{m}(38)$ dimethacrylate, Hydrophilic aliphatic dimethacrylate

Paste B: Bis-GMA,

Hydrophobic aromatic dimethacrylate, Hydrophilic aliphatic dimethacrylate

\begin{tabular}{lcc}
\hline MDP solution & & \\
& 10-Methacryloyloxydecyl dihydrogen phosphate (MDP), ethanol & Kuraray Noritake Dental \\
\hline Silane solution & $\gamma$-Methacryloxypropyltrimethoxysilane ( $\gamma$-MPTS), ethanol & $\begin{array}{l}\text { Sigma-Aldrich, St.Louis, } \\
\text { MO, USA }\end{array}$ \\
\hline Acetic acid solution & & Nacalai tesque, \\
& Acetic acid, ethanol & Kyoto, Japan \\
\hline Phosphoric Acid & & Kuraray Noritake Dental \\
\hline K-etchant gel & $40 \%$ phosphoric acid &
\end{tabular}

were cross-sectioned by argon ion milling (SM-090101 Cross-Section Polisher, JEOL). After a thin layer of carbon was vaporized on the surface (JEE-420T Vacuum Evaporators, JEOL), the specimens were examined using a field emission-gun SEM (Feg-SEM; JSM-6701F, JEOL), operated at $5 \mathrm{kV}$ and using an annular semiconductor detector.

Scanning transmission electron microscopy (STEM) and energy dispersive X-ray spectroscopy (EDS)

STEM specimens were prepared by argon ion milling technique using an ion slicer (EM-09100IS, JEOL). The blocks were cut and polished for argon ion milling preprocessing. STEM was performed using a $200-\mathrm{kV}$ STEM system (JEM-2100F, JEOL). JEM-2100F was equipped with a probe-forming $\mathrm{Cs}$ corrector (CEOS, Heidelberg, Germany), STEM bright-field (BF), annular dark-field (ADF) detectors, and EDS (JED-2300T, JEOL) spectrometer attachments. The probe-forming Cs corrector enabled sub-Angstrom-resolution STEM imaging (minimum probe size: $0.09 \mathrm{~nm}$ ).

\section{Microtensile bond strength ( $\mu$ TBS) measurement}

Figure 1 illustrates how the specimens were fabricated in this study. Specimens from each CAD/CAM block were randomly divided into seven groups (12 specimens per group) according to surface treatment method (Fig. 2).

A CAD/CAM block was cut into half to yield two square-block specimens. The adhesive surfaces were polished with diamond wrap film (3M, St. Paul, MN, USA) under water irrigation, and plasma-irradiated using a plasma equipment (P500-SM, SAKIGAKE, Kyoto, Japan) for $60 \mathrm{~s}$ with the nozzle positioned at 10 


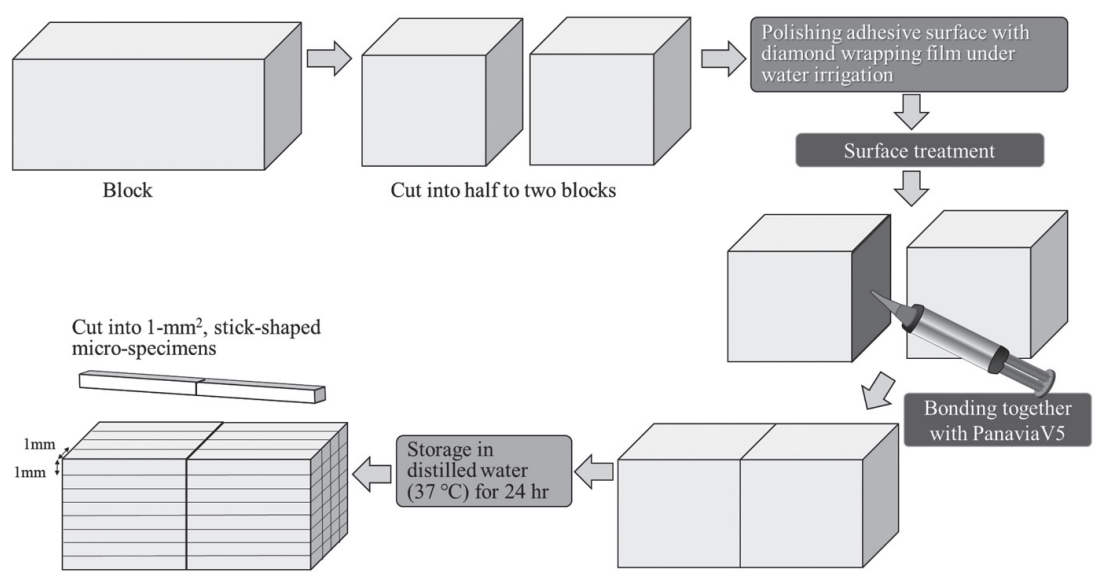

Fig. 1 Schematic illustration of specimens fabrication.

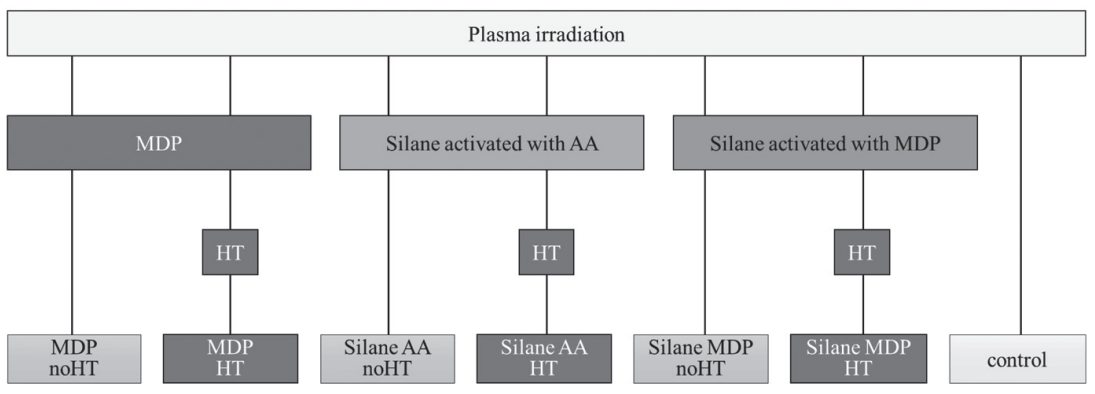

Fig. 2 Schematic diagram for surface treatments. MDP: 10-methacryloyloxydecyl dihydrogen phosphate, AA: Acetic acid, HT: Heat treatment

$\mathrm{mm}$ from the specimen surface. These prepared surfaces were subjected to one or a combination of the following surface treatment methods:

1) MDP: Solution of $2 \mathrm{wt} \%$ of MDP in ethanol (Kuraray Noritake Dental, Tokyo, Japan) was applied using a microbrush for $2 \mathrm{~min}$ and dried with oil-free air for $30 \mathrm{~s}$.

2) Silane AA: Solution of $2 \mathrm{wt} \%$ of $\gamma$-MPTS in ethanol (Sigma-Aldrich, St. Louis, MO, USA) was activated immediately before use with $2 \mathrm{wt} \%$ of acetic acid (AA; Nacalai Tesque, Kyoto, Japan), applied for $2 \mathrm{~min}$ and air-dried for $30 \mathrm{~s}$.

3) Silane MDP: Solution $2 \mathrm{wt} \%$ of $\gamma$-MPTS in ethanol was activated immediately before use with $2 \mathrm{wt} \%$ of MDP, applied for $2 \mathrm{~min}$ and air-dried for $30 \mathrm{~s}$.

4) HT: Heat treatment was carried at $110^{\circ} \mathrm{C}$ for 5 $\min$.

5) Control: Without any surface or heat treatment.

Specimens subjected to the same surface treatment were bonded together with Panavia V5 (Kuraray Noritake Dental), where a $10 \mathrm{~N}$ load was applied and maintained for $3 \mathrm{~min}$. After 24 -h storage in $37^{\circ} \mathrm{C}$ distilled water, the specimens were cut into $1-\mathrm{mm}^{2}$, stick-shaped micro-specimens with the aid of a $0.3-\mathrm{mm}$ diamond cutoff wheel (Struers) mounted in an Accutom-50 cut-off machine (Struers).
The micro-specimens were fixed to a jig using a cyanoacrylate glue (Model Repair II Blue, DentsplySankin, Ohtawara, Japan), and stressed at a crosshead speed of $1 \mathrm{~mm} / \mathrm{min}$ until failure using a universal testing device (Micro Tensile Tester, BISCO, Chicago, IL, USA) equipped with a load cell of $100 \mathrm{~N}$.

The data were subjected to Levene test to evaluate homogeneity of variance $(p<0.05)$, and statistically evaluated by Kruskal-Wallis test and Steel-Dwasss multiple comparison test. The significance level was set at $5 \%$.

\section{Phosphate group adsorption detection}

To detect phosphate group adsorption on the blocks, Attenuated Total Reflection (ATR)-FTIR spectroscopy (Bruker Vertex 70, Ettlingen, Germany) was carried out on both CAD/CAM blocks with and without the application of $40 \%$ phosphoric acid solution (PA: K-etchant gel, Kuraray Noritake Dental). FTIR spectra were recorded after 64 successive scans and at $4 \mathrm{~cm}^{-1}$ spectral resolution. Each sample was measured three times.

$\mu$ TBS values were also measured for Silane MDP HT and Silane AA HT in each block, with and without PA application. After plasma treatment, the adhesive surface was pretreated with PA for $60 \mathrm{~s}$, washed with 
distilled water, and air-dried for $30 \mathrm{~s}$. Then the different surface treatments, the fabrication of specimens, and $\mu$ TBS measurements were performed as described above.

\section{RESULTS}

\section{SEM findings}

Figure 3 shows the SEM images. Inorganic filler material in PIC (a) revealed a polygonal shape with a particle size of about $5 \mu \mathrm{m}$. In MFR (b), a polygonal or spherical shape was observed with a particle size of
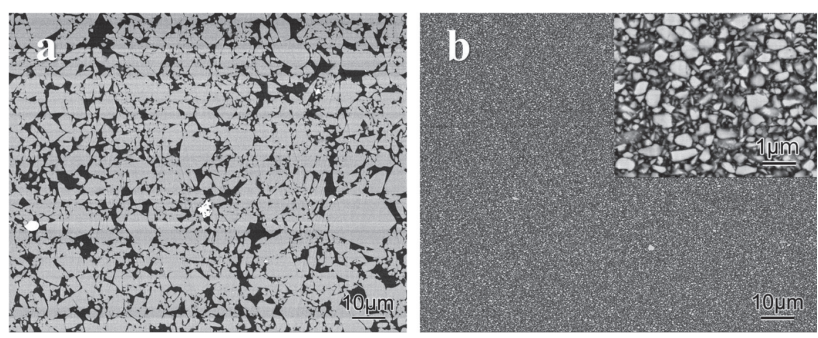

Fig. 3 SEM images.

a: PIC (Polymer-infiltrated ceramic); b: MFR (Microfilled resin). (a)

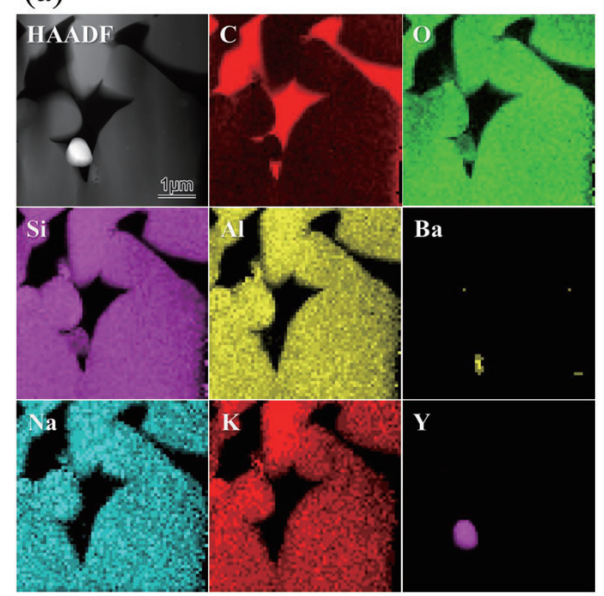

(b)

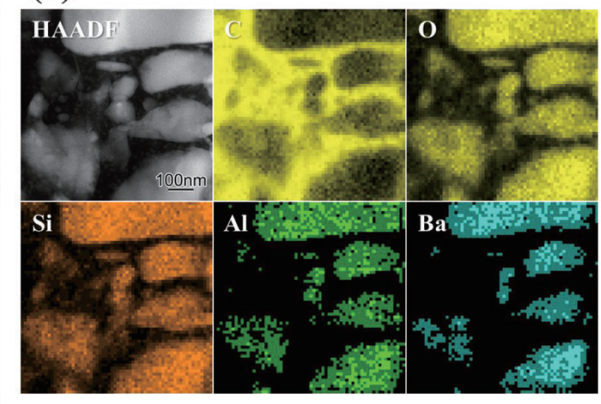

Fig. 4 High-angle annular dark-field (HAADF) STEM photomicrographs with EDS mapping images of PIC (a) and MFR (b).

EDS mapping revealed the distributions of carbon (C), oxygen (O), silicon ( $\mathrm{Si}$ ), aluminium (Al), barium $(\mathrm{Ba})$, sodium $(\mathrm{Na})$, potassium $(\mathrm{K})$ and yttrium $(\mathrm{Y})$.

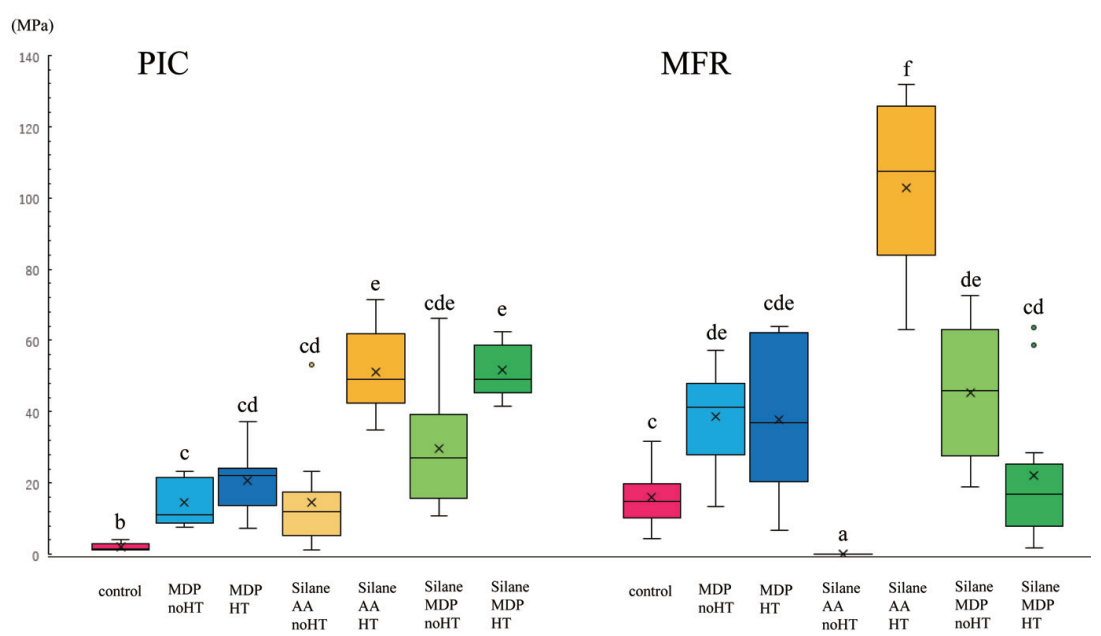

Fig. 5 Median values of $\mu$ TBS (MPa) of each treatment group.

PIC: Polymer-infiltrated ceramic; MFR: Microfilled resin; MDP:10methacryloyloxydecyl dihydrogen phosphate; AA: Acetic acid; HT: Heat treatment. Values with same superscripts $(\mathrm{a}-\mathrm{f})$ in each column are not significantly different $(p>0.05)$ according to Kruskal-Wallis test and Steel-Dwass multiple comparison test. 
Table 2 Median values of $\mu$ TBS (MPa) after PA application to Silane MDP HT and Silane AA HT

\begin{tabular}{ccccc}
\hline & \multicolumn{4}{c}{ Treatment } \\
\cline { 2 - 5 } & Silane AA HT & PA+Silane AA HT & Silane MDP HT & PA+Silane MDP HT \\
\hline PIC & $49.2(34.9-71.6)^{\mathrm{c}}$ & $66.2(19.7-103.8)^{\mathrm{c}}$ & $49.2(41.6-62.6)^{\mathrm{bc}}$ & $68.0(51.9-76.0)^{\mathrm{c}}$ \\
MFR & $107.4(63.1-131.9)^{\mathrm{d}}$ & $0.0^{\mathrm{a}}$ & $17.0(1.8-63.5)^{\mathrm{b}}$ & $36.0(8.5-80.9)^{\mathrm{bc}}$ \\
\hline
\end{tabular}

PIC: polymer infiltrated ceramics, MFR: microfilled resins, PA: phosphoric acid, AA: acetic acid, MDP: 10-methacryloyloxydecyl dihydrogen phosphate, HT: heat treatment

Values with same superscripts (a-d) in each column are not significantly different $(p>0.05)$ according to Kruskal-Wallis test and Steel-Dwass multiple comparison test.

about $0.2 \mu \mathrm{m}$. The ratio of inorganic component was higher in PIC than in MFR.

\section{STEM and EDS findings}

Figure 4 shows the STEM and EDS images. The inorganic elements of PIC (a) were identified as C, O, $\mathrm{Si}, \mathrm{Al}, \mathrm{Ba}, \mathrm{Na}, \mathrm{K}$ and $\mathrm{Y}$, while those of MFR (b) were C, $\mathrm{O}, \mathrm{Si}, \mathrm{Al}, \mathrm{Ba}$.

\section{$\mu T B S$ values}

Figure 5 and Table 2 show the $\mu$ TBS values. The $\mu$ TBS value (Median (Min-Max) MPa) of the control group of MFR (14.9 (4.5-31.8) MPa) was higher than that of PIC (1.6 (1.3-4.0) MPa).

In PIC and MFR, the $\mu$ TBS value of the MDP no HT (PIC: 11.2 (7.6-23.3) MPa, MFR: 41.2 (13.4-57.2) MPa) ware significantly higher than the control $(p<0.05)$. On the other hand, there were no significant differences between MDP noHT and MDP HT (PIC: 22.2 (7.2-37.1) MPa, MFR: 36.9 (6.7-64.0) MPa). For MDP noHT, the $\mu$ TBS value of MFR was significantly higher than that of PIC $(p<0.05)$.

In PIC, Silane AA noHT showed 11.9 (1.3-53.2) $\mathrm{MPa}$, which was significantly higher than the control $(p<0.05)$. Moreover, Silane AA HT (49.2 (34.9-71.6) $\mathrm{MPa})$ showed a significantly higher value than Silane AA noHT $(p<0.05)$. In MFR, all specimens of the Silane AA noHT group debonded before test and showed $0 \mathrm{MPa}$. On the other hand, Silane AA HT showed the highest value (107.4 (63.1-131.9) MPa) when compared with all the other groups.

In PIC, there was not significantly different between Silane MDP noHT (27.1 (10.7-66.2) MPa) and Silane MDP HT (49.2 (41.6-62.6) MPa). These values were also significantly higher than the control $(p<0.05)$. However, Silane MDP noHT was not higher than MDP noHT or Silane AA noHT. Silane MDP HT and Silane AA HT showed the highest and similar values.

In MFR, there was not significantly different between Silane MDP noHT (46.1 (18.8-72.5) MPa) and Silane MDP HT (17.0 (1.8-63.5) MPa). Silane MDP noHT showed a significantly higher value than the control $(p<0.05)$. Silane MDP noHT, MDP noHT and MDP HT showed similar values.

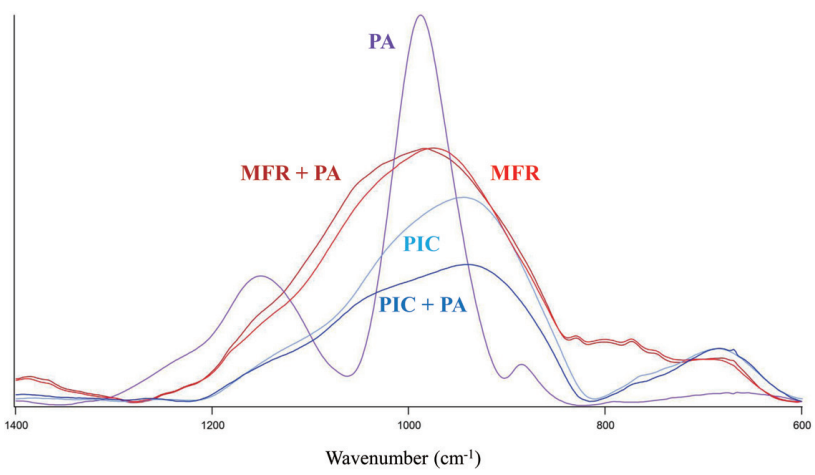

Fig. 6 FTIR spectra of PA (40\% phosphoric acid aqueous solution), PIC (polymer-infiltrated ceramic) and MFR (Microfilled resin) with and without PA application.

\section{Phosphate group adsorption}

Figure 6 shows the FTIR spectra. The MFR peak was shifted to a higher wavenumber according to the phosphate peak when PA was applied. For PIC, its peak did not show the shift but a decrease in strength instead.

Table 2 shows the $\mu$ TBS results of Silane MDP HT and Silane AA HT with and without PA application. In PIC, there were no significant changes in $\mu$ TBS through the PA application. Moreover, PA+Silane AA HT and PA+Silane MDP HT showed similar values. In MFR, the $\mu$ TBS value of Silane AA HT dramatically decreased with PA application, showing $0 \mathrm{MPa}$. The bond strength of Silane MDP HT showed a similar tendency as PIC, in that there was no significant change after PA application.

\section{DISCUSSION}

Two new CAD/CAM composite blocks, PIC and MFR, were used in this study. PIC consisted of a porous ceramic network infiltrated with a methacrylate polymer, and the proportion of inorganic filler material was $86 \%$ by mass. MFR consisted of nanosized inorganic clusters filled into a resin matrix at high density, and the proportion of inorganic filler material was $71 \%$ by mass. SEM revealed that the inorganic material of PIC exhibited a polygonal shape with an average size of about $5 \mu \mathrm{m}$. In MFR, the 
inorganic material exhibited a polygonal or spherical shape with an average particle size of about $0.2 \mu \mathrm{m}$. SEM also revealed that the inorganic filler material of PIC occupied a larger surface area than in MFR. Through STEM, PIC and MFR were observed to have different inorganic filler compositions. PIC consisted of $\mathrm{C}, \mathrm{O}, \mathrm{Si}$, $\mathrm{Al}, \mathrm{Ba}, \mathrm{Na}, \mathrm{K}$, and $\mathrm{Y}$, while MFR consisted of $\mathrm{C}, \mathrm{O}, \mathrm{Si}$, $\mathrm{Al}$, and $\mathrm{Ba}$.

On $\mu$ TBS, the control groups of both CAD/ CAM blocks revealed the difference in their bonding performance to luting agent. MFR's control had a $\mu$ TBS value about 9 times higher than that of PIC. It is difficult for methacrylic acid monomers to bond to inorganic fillers ${ }^{19}$. Hence, it was easier for the methacrylic acid monomers in the luting agent to form a stronger bond to MFR than to PIC, hence accounting for MFR's markedly higher $\mu$ TBS value. The inorganic elements in the CAD/ CAM blocks were coated with a silane coupling agent to be bonded to the matrix resin ${ }^{26}$. Due to the structural features, the inorganic fillers in MFR were more exposed than those in PIC; hence, the maximum $\mu$ TBS value of PIC was approximately half of MFR's. The mechanical properties of each block's material might also account for the markedly different maximum $\mu$ TBS values: PIC had lower flexural strength and higher elastic modulus than $\mathrm{MFR}^{5-7)}$.

Treatment with MDP alone improved the bonding performance of both CAD/CAM blocks to luting agents. MDP treatment groups showed higher bond strength: they were about 7 times higher than the control group for PIC and about 2 times higher for MFR. HT after MDP application did not offer increased bond strength to both blocks. However, a survey of published literature revealed that there were no reports on the effect of MDP treatment alone for PIC and MFR. In one study, a universal adhesive containing MDP significantly increased the shear bond strength of PIC from $7.715 \pm 2.336 \mathrm{MPa}$ to $10.728 \pm 3.449 \mathrm{MPa}^{25)}$. Contrastingly, a silane coupling primer containing MDP did not increase the shear bond strength of mirror-polished PIC and MFR (PIC: Control-0.00 MPa, Primer-0.20 MPa; MFR: Control-0.00 MPa, Primer-0.00 MPa ${ }^{24)}$. In another study, hydrofluoric acid etching or air abrasion followed by application of universal adhesive containing MDP and silane coupling agent significantly increased the four-point bending strengths of PIC and MFR to luting agent than hydrofluoric acid etching alone, air abrasion alone or control with no pretreatments ${ }^{27}$.

MDP, an acidic functional monomer, has a hydrophilic phosphate group at one end of the molecule and which bonds strongly with metal oxides, and a hydrophobic vinyl group at the other end which reacts with monomers ${ }^{15,16)}$. A study had observed through NMR spectroscopy that the phosphate group formed hydrogen bonds and ionic bonds to hydroxyl groups and zirconium on the surface of zirconia ${ }^{28}$. In this study, the phosphate group of MDP seemed to be first bonded to the hydroxyl group or the oxides in the inorganic filler material, then to the luting agent. The chemical bond between MDP and zirconia depends on the number of
$\mathrm{H}^{+}$ions, and it is easy to form bonds in alkaline, neutral and acidic conditions ${ }^{29}$. Hence, the chemical properties of the adhesive surface of each block used in this study might have affected the bonding performance to luting agent. In general, heat treatment changes the chemical equilibrium and reaction rate; hence, it is sometimes used to improve the adhesive surfaces ${ }^{23,30}$. However in this study, heat treatment at $110^{\circ} \mathrm{C}$ for 5 min after MDP application did not improve the bonding of both CAD/CAM blocks. Similarly, it was reported that heat treatment after application of a universal primer containing MDP and silane coupling agent did not significantly change the shear bond strength of zirconia ${ }^{311}$.

$\gamma$-MPTS used in this study had functional groups which bonds to both inorganic elements and luting agents. Methoxy group is activated by a hydrolysis catalyst in an acidic medium to form silanol, which then produces a strong bond called siloxane bonding on the surface of inorganic filler materials (especially silica, glass and quartz) to mediate adhesion to luting agent ${ }^{32}$.

In PIC, AA-activated silane coupling agent increased the $\mu$ TBS value by about 7 times higher than the control, a result similar to that by MDP alone. In MFR, Silane AA noHT showed $0 \mathrm{MPa}$ - an evident case of inhibited adhesion. Differences in the composition and surface structure of their inorganic components could have affected the effectiveness of silane coupling agent. For PIC, composition of the inorganic filler material was similar to other conventional glass ceramic materials, which thus enhanced the effectiveness of silane coupling agent $t^{6}$. In addition, the particle size and occupied area of the inorganic filler material on the surface of PIC were vastly different from those of MFR.

AA-activated silane coupling agent followed by HT increased the $\mu$ TBS values of both PIC and MFR. Notably, MFR's Silane AA HT group showed 107.4 (63.1-131.9) $\mathrm{MPa}$ - the highest value among all the groups. The hydrolysis reaction of silane coupling agents is influenced by temperature ${ }^{31}$. The heat treatment after silane coupling agent application not only helps to remove water, alcohol and other reaction byproducts, it also helps to complete the condensation reaction for siloxane bond formation ${ }^{33)}$. Hence, it was reported that heat treatment at $100^{\circ} \mathrm{C}$ for $5 \mathrm{~min}$ after silane coupling agent application improved the $\mu$ TBS between lithium disilicate ceramic and composite resin ${ }^{23)}$.

The composition, occupied area and shape of inorganic material on the adhesive surface of PIC seemed to favor the function of the silane coupling agent. Conversely, the nano-fillers of MFR's inorganic material might have less hydroxy groups and metal oxides on the surface to form scaffolds as sites for chemical bonding. The region where a silane coupling agent exists between an inorganic substrate and the resin is a continuously changing interfacial phase called the "interphase" ${ }^{34)}$. The interphase consists of both chemically bonded silanes (chemisorbed silanes) and physically adsorbed silanes (physisorbed silanes), and the latter are known to inhibit interfacial adhesion. Hence, the composition and 
properties of the interphase are also pivotal to bonding effectiveness.

In PIC, the bond strength of Silane MDP noHT reflected the synergistic effect of both MDP and Silane. In MFR, MDP-activated silane coupling agent showed a significantly higher $\mu$ TBS value than the control, but the result was similar to that by MDP alone. It is noteworthy that AA-activated silane coupling agent showed $0 \mathrm{MPa}$, which meant that silane coupling agents might deteriorate adhesion to MFR. On the bonding of CAD/CAM composite blocks to luting agent, different studies have yielded varying results. On the one hand, shear bond strength was not significantly increased by the use of universal adhesive containing MDP and silane coupling agent ${ }^{24}$. On the other hand, an adhesive primer containing MDP and silane coupling agent significantly increased the shear bond strength between luting agent and $\mathrm{PIC}^{25)}$. When used after hydrofluoric acid or air abrasion treatment, a primer containing MDP and silane coupling agent significantly improved the four-point bending strength, versus treated by acid or air abrasion alone ${ }^{27}$. Similarly with lithium disilicate ceramic, MDP was superior to acetic acid when used as a hydrolysis catalyst for silane coupling agent by yielding significantly higher bond strength ${ }^{21,22)}$.

Concerning heat treatment silane coupling agent with MDP, $\mu$ TBS between PIC and luting agent was reported to be improved by a combined treatment of MDP-containing silane coupling agent followed by HT at $100^{\circ} \mathrm{C}$ for $2 \mathrm{~min}^{30)}$. In PIC, HT promoted hydrolysis and formation of siloxane bonds for both AA-activated silane coupling agent and MDP-activated silane coupling agent $^{32,33)}$, thereby also promoting bonding to luting agent. These phenomena were also observed in this study which the $\mu$ TBS in PIC increased about 1.8 times after HT. But in MFR, HT tended to reduce the bond strength achieved by MDP-activated silane coupling agent. The difference between PIC and MFR in the case of Silane MDP HT could be attributed to the adsorption performance of MDP and silane coupling agent to the inorganic component. Larger molecular weight of MDP to $\gamma$-MPTS could cause the interphase which existed in the presence of both MDP and $\gamma$-MPTS to become thicker than $\gamma$-MPTS alone. A thicker interphase would result in a higher proportion of physically adsorbed silanes. Eventually, condensation and oligomerisation of $\gamma$-MPTS by heating might increase the polymer film thickness on the surface ${ }^{20,22,32,35)}$, which then weakened the bond strength.

The higher adsorption of the phosphate group to the inorganic components of MFR was revealed by FTIR, which further explained the difference in bonding results between PIC and MFR. In PIC, there was a decrease in peak strength and an increase in $\mu$ TBS, which could be caused by a change in surface properties induced by PA application. On $\mu$ TBS, PIC's value was only slightly increased after PA application. In MFR, PA only slightly increased the $\mu$ TBS value in Silane MDP HT, but caused a significant decrease to $0 \mathrm{MPa}$ in Silane AA HT. MFR showed high adsorption capacity for the phosphate groups, and PA application could make subsequent silane coupling agent binding difficulty.

PA has been generally used for the pretreatment of adhesive surfaces. It improves adhesive strength by rendering a cleaning effect on the adhesive surface ${ }^{13)}$, thereby reducing the contact angle and enhancing the function of silane coupling agents. It has also been reported that PA slightly increased the surface roughness of PIC, but had only a slight effect on $\mathrm{MFR}^{36)}$. Nonetheless, the phosphate groups might remain on the CAD/CAM hybrid resin surface. These adsorbed phosphate groups then reduced the bonding effectiveness of the adhesives.

\section{CONCLUSIONS}

Within the limitations of this study, the following conclusions were drawn:

(1) The initial bonding of PIC to luting agent could be improved by surface treatment with MDP or silane coupling agent, which could be further improved with succeeding heat treatment.

(2) The initial adhesiveness of MFR could be deteriorated by silane coupling agent treatment without heat processing. MFR possessed higher adsorption capacity for the phosphate group, resulting in improved bonding with MDP.

\section{ACKNOWLEDGMENTS}

This work was partially supported by a Grant-in-aid for Scientific Research (KAKENHI), Grant Number 17K11749, from the Japan Society for the Promotion of Science (JSPS).

\section{REFERENCES}

1) Miyazaki T, Hotta Y, Kunii J, Kuriyama S, Tamaki Y. A review of dental CAD/CAM: current status and future perspectives from 20 years of experience. Dent Mater J 2009; 28: 44-56.

2) Patzelt SB, Spies BC, Kohal RJ. CAD/CAM-fabricated implant-supported restorations: a systematic review. Clin Oral Implants Res 2015; 26: 77-85.

3) Ludovichetti FS, Trindade FZ, Werner A, Kleverlaan CJ, Fonseca RG. Wear resistance and abrasiveness of CAD-CAM monolithic materials. J Prosthet Dent 2018; 120: 318 e1-8.

4) Nguyen JF, Migonney V, Ruse ND, Sadoun M. Resin composite blocks via high-pressure high-temperature polymerization. Dent Mater 2012; 28: 529-534.

5) Lawson NC, Bansal R, Burgess JO. Wear, strength, modulus and hardness of CAD/CAM restorative materials. Dent Mater 2016; 32: e275-283.

6) Sonmez N, Gultekin P, Turp V, Akgungor G, Sen D, Mijiritsky E. Evaluation of five CAD/CAM materials by microstructural characterization and mechanical tests: a comparative in vitro study. BMC Oral Health 2018; 18: 5.

7) Furtado de Mendonca A, Shahmoradi M, Gouvêa CVD, De Souza GM, Ellakwa A. Microstructural and mechanical characterization of CAD/CAM materials for monolithic dental restorations. J Prosthodont 2019; 28: e587-594.

8) Santing HJ, Kleverlaan CJ, Werner A, Feilzer AJ, Raghoebar GM, Meijer HJ. Occlusal wear of provisional implantsupported restorations. Clin Implant Dent Relat Res 2015; 
17: 179-185.

9) He LH, Swain M. A novel polymer infiltrated ceramic dental material. Dent Mater 2011; 27: 527-534.

10) Tekçe N, Fidan S, Tuncer S, Kara D, Demirci M. The effect of glazing and aging on the surface properties of CAD/CAM resin blocks. J Adv Prosthodont 2018; 10: 50-57.

11) Costa AK, Kelly RD, Fleming GJ, Borges AL, Addison O. Laminated ceramics with elastic interfaces: a mechanical advantage? J Dent 2015; 43: 335-341.

12) Reymus M, Roos M, Eichberger M, Edelhoff D, Hickel R, Stawarczyk B. Bonding to new CAD/CAM resin composites: influence of air abrasion and conditioning agents as pretreatment strategy. Clin Oral Investig 2019; 23: 529-538.

13) Campos F, Almeida CS, Rippe MP, de Melo RM, Valandro LF, Bottino MA. Resin bonding to a hybrid ceramic: effects of surface treatments and aging. Oper Dent 2016; 41: 171-178.

14) Güngör MB, Nemli SK, Bal BT, Ünver S, Doğan A. Effect of surface treatments on shear bond strength of resin composite bonded to $\mathrm{CAD} / \mathrm{CAM}$ resin-ceramic hybrid materials. J Adv Prosthodont 2016; 8: 259-266.

15) De-Paula DM, Loguercio AD, Reis A, Frota NM, Melo R, Yoshihara K, et al. Micro-Raman vibrational identification of 10-MDP bond to zirconia and shear bond strength analysis. Biomed Res Int 2017: 8756396.

16) Fujita K, Ma S, Aida M, Maeda T, Ikemi T, Hirata M, et al. Effect of reacted acidic monomer with calcium on bonding performance. J Dent Res 2011; 90: 607-612.

17) Chen Y, Lu Z, Qian M, Zhang H, Chen C, Xie H, et al. Chemical affinity of 10-methacryloyloxydecyl dihydrogen phosphate to dental zirconia: Effects of molecular structure and solvents. Dent Mater 2017; 33: e415-427.

18) Yazdi FM, Moosavi H, Atai M, Zeynali M. Dentin bond strength and degree of conversion evaluation of experimental self-etch adhesive systems. J Clin Exp Dent 2015; 7: e243249.

19) Lung CY, Matinlinna JP. Aspects of silane coupling agents and surface conditioning in dentistry: an overview. Dent Mater 2012; 28: 467-477.

20) Yoshihara K, Nagaoka N, Sonoda A, Maruo Y, Makita Y, Okihara T, et.al. Effectiveness and stability of silane coupling agent incorporated in 'universal' adhesives. Dent Mater 2016; 32: 1218-1225.

21) Tanimura H. Effect of the type of acidic monomer on the bond durability of two-bottle type ceramic primer. J Oral Sci 2017; 59: 131-137.

22) Maruo Y, Nishigawa G, Yoshihara K, Minagi S, Matsumoto T, Irie M. Does 8-methacryloxyoctyl trimethoxy silane (8-MOTS) improve initial bond strength on lithium disilicate glass ceramic? Dent Mater 2017; 33: e95-100.

23) Abduljabbar T, AlQahtani MA, Jeaidi ZA, Vohra F. Influence of silane and heated silane on the bond strength of lithium disilicate ceramics — An in vitro study. Pak J Med Sci 2016; 32: 550-554.

24) Demirtag Z, Culhaoglu AK. Surface roughness of ceramicresin composites after femtosecond laser irradiation, sandblasting or acid etching and their bond strength with and without silanization to a resin cement. Oper Dent 2019; 44: 156-167

25) Barutcigil K, Barutcigil Ç, Kul E, Özarslan MM, Buyukkaplan US. Effect of different surface treatments on bond strength of resin cement to a CAD/CAM restorative material. J Prosthodont 2019; 28: 71-78.

26) Nihei T. Dental applications for silane coupling agents. J Oral Sci 2016; 58: 151-155.

27) Kömürcüoğlu MB, Sağırkaya E, Tulga A. Influence of different surface treatments on bond strength of novel CAD/CAM restorative materials to resin cement. J Adv Prosthodont 2017; 9: 439-446.

28) Nagaoka N, Yoshihara K, Feitosa VP, Tamada Y, Irie M, Yoshida Y, et al. Chemical interaction mechanism of 10-MDP with zirconia. Sci Rep 2017; 7: 455-463

29) Xie H, Tay FR, Zhang F, Lu Y, Shen S, Chen C. Coupling of 10-methacryloyloxydecyldihydrogenphosphate to tetragonal zirconia: Effect of $\mathrm{pH}$ reaction conditions on coordinate bonding. Dent Mater 2015; 31: e218-225.

30) de Carvalho RF, Cotes C, Kimpara ET, Leite FP, Özcan M. Heat treatment of pre-hydrolyzed silane increases adhesion of phosphate monomer-based resin cement to glass ceramic. Braz Dent J 2015; 26: 44-49.

31) Dal Piva AMO, Carvalho RLA, Lima AL, Bottino MA, Melo RM, Valandro LF. Silica coating followed by heat-treatment of MDP-primer for resin bond stability to yttria-stabilized zirconia polycrystals. J Biomed Mater Res B Appl Biomater 2019; 107: 104-111.

32) Matinlinna JP, Lung CYK, Tsoi JKH. Silane adhesion mechanism in dental applications and surface treatments: A review. Dent Mater 2018; 34:13-28.

33) Shen Chiayi, Oh Won-suck, Williams James R. Effect of post-silanization drying on the bond strength of composite to ceramic. J Prosthet Dent 2004; 91: 453-458

34) Wilson KS, Allen AJ, Washburn NR, Antonucci JM. Interphase effects in dental nanocomposites investigated by small-angle neutron scattering. J Biomed Mater Res A 2007; 81: 113-123.

35) Yao C, Yu J, Wang Y, Tang C, Huang C. Acidic pH weakens the bonding effectiveness of silane contained in universal adhesives. Dent Mater 2018; 34: 809-818

36) Strasser T, Preis V, Behr M, Rosentritt M. Roughness, surface energy, and superficial damages of CAD/CAM materials after surface treatment. Clin Oral Investig 2018; 22: 2787-2797. 